\title{
Covariation in individuals' sensitivities to bitter compounds: Evidence supporting multiple receptor/transduction mechanisms
}

\author{
JEANNINE F. DELWICHE \\ Ohio State University, Columbus, Ohio \\ and Monell Chemical Senses Center, Philadelphia, Pennsylvania \\ and \\ ZIVJENA BULETIC and PAUL A. S. BRESLIN \\ Monell Chemical Senses Center, Philadelphia, Pennsylvania
}

\begin{abstract}
People vary widely in their sensitivities to bitter compounds, but the intercorrelation of these sensitivities is unknown. Our goal was to investigate correlations as a function of individual sensitivities to several bitter compounds representative of different chemical classes and, from these correlations, infer the number and variety of potential bitterness transduction systems for these compounds. Twenty-six subjects rated and ranked quinine $\mathrm{HCl}$, caffeine, (-)-epicatechin, tetralone, L-phenylalanine, L-tryptophan, magnesium sulfate, urea, sucrose octaacetate (SOA), denatonium benzoate, and $n$ propylthiouracil (PROP) for bitterness. By examining individual differences, ratings and rankings could be grouped into two general clusters-urea/phenylalanine/tryptophan/epicatechin, and quinine/caffeine/SOA/denatonium benzoate/tetralone/magnesium sulfate-none of which contained PROP. When subjects were grouped into the extremes of sensitivity to PROP, a significant difference was found in the bitterness ratings, but not in the rankings. Therefore, there are also subjects who possess diminished absolute sensitivity to bitter stimuli but do not differ from other subjects in their relative sensitivities to these compounds.
\end{abstract}

The diversity of compounds that elicit bitter-taste sensations is vast, including both inorganic and organic compounds (Belitz \& Wieser, 1985; Spielman, Huque, Whitney, \& Brand, 1992). Compounds with bitter tastes have been categorized as amines (e.g., denatonium), ureas/thioureas, amino acids (such as L-phenylalanine, Ltryptophan, L-arginine, etc.), alkaloids (including caffeine and quinine), acetylated sugars (e.g., sucrose octaacetate $[\mathrm{SOA}]$ ), isohumulones (e.g., isohumulone, iso-cohumulone, tetrahydroisohumulone, etc.), phenols (including flavanols catechin and epicatechin), carbamates (e.g., phenylthiocarbamide [PTC]), and ionic salts (e.g., magnesium sulfate) (Belitz \& Wieser, 1985; Spielman et al., 1992). It is unlikely that a single bitter receptor can account for sensitivity to all these classes of compounds (Brand, 1997; Spielman et al., 1992). The goal of the present paper was to determine whether classes of bitter transduction processes in the general population

This work was funded by NIH Grants R29 DC02995 (to P.A.S.B) and F32 DC00384-02 (to J.F.D). The authors thank Alexander A. Bachmanov, Gary K. Beauchamp, Joseph G. Brand, Bruce Schneider, David V. Smith, and the anonymous reviewers for their thoughtful comments on earlier versions of this manuscript. Correspondence should be addressed to P. A. S. Breslin, Monell Chemical Senses Center, 3500 Market St., Philadelphia, PA 19104 (e-mail: breslin@monell.org). might be revealed by examining and correlating individual differences in sensitivities to bitter compounds.

Large individual differences in the bitterness of these compounds have been well documented. The best known variation among subjects' sensitivities to bitter chemicals is found for the perception of $n$-propylthiouracil (PROP), PTC, and other antithyroid compounds that contain the $\mathrm{N}-\mathrm{C}=\mathrm{S}$ chemical group (e.g., Barnicot, Harris, \& Kalmus, 1951; Bartoshuk, Duffy, Reed, \& Williams, 1996; Fischer \& Griffin, 1963; Hall, Bartoshuk, Cain, \& Stevens, 1975; Harris \& Kalmus, 1949; Kemp \& Birch, 1992; Thorngate, 1997; Yokomukai, Cowart, \& Beauchamp, 1993), although wide-ranging responses across individuals to other bitter compounds have been found as well (Yokomukai et al., 1993). How strongly one perceives a particular concentration of PROP has not proven to be a reliable indicator of how strongly one perceives particular concentrations of many other bitter substances, including quinine, caffeine, and urea, despite numerous attempts to demonstrate a predictive relationship (Bartoshuk, 1979; Hall et al., 1975; Lawless, 1979; Leach \& Noble, 1986; Mela, 1989; Schifferstein \& Frijters, 1991; Yokomukai et al., 1993). Although sensitivity to PROP and sensitivity to PTC do not correlate with sensitivity to other bitter compounds, the possibility remains that sensitivity to other bitter compounds will correlate with each other. 
The substantial independent variation in sensitivities to the many bitter-tasting compounds suggests that there is more than one physiological transduction mechanism for the perception of bitter tastes. Dahl, Erickson, and Simon (1997) attempted to infer the idiosyncratic distribution of transduction mechanisms for bitter taste stimuli in rat taste receptor cells via primary neural recordings. They recorded single neuron responses to 10 bitter-tasting compounds from rat glossopharyngeal and chorda tympani nerves and summarized their differences in evoked responses in multidimensional scaling space. They found evidence that first-order gustatory neurons respond differentially to the bitter stimuli and inferred that bitter-sensing taste receptor cells comprise a heterogeneous population.

By using a strategy similar to that of Dahl et al. (1997) and determining whether compounds cluster together in humans as a function of sensitivities across individuals, it may be possible to infer the number and variety of potential bitterness transduction systems for the compounds tested. Yokomukai et al. (1993) found that sensitivity to quinine sulfate and sensitivity to urea were unrelated, but individuals more sensitive to quinine than to urea tended to find the bitterness of suprathreshold caffeine and sucrose octaacetate to be greater than that of suprathreshold magnesium sulfate, whereas individuals more sensitive to urea than to quinine showed the reverse pattern. Consistent with these findings, McBurney, Smith, and Shick (1972) found that adaptation to $100 \mu \mathrm{M}$ quinine hydrochloride significantly decreased the perceived bitterness of $1 \mathrm{mM}$ SOA and $10 \mathrm{mM}$ caffeine, but not $250 \mathrm{mM}$ magnesium sulfate, whereas adaptation to $1.0 \mathrm{M}$ urea significantly decreased the perceived bitterness of $250 \mathrm{mM}$ magnesium sulfate, but not $1 \mathrm{mM}$ SOA and $10 \mathrm{mM}$ caffeine. Lawless (1979) also confirmed these findings for quinine, urea, and creatine.

Recently, Adler et al. (2000) and Matsunami, Montmayeur, and Buck (2000) have identified a novel family of 40-80 putative $\mathrm{G}$ protein-coupled bitter receptors (Tas2rs) that are expressed in selected taste receptor cells $(\sim 15 \%)$, further suggesting bitter receptor heterogeneity. The genes are organized into serial, head-to-tail clusters on human chromosomes 5, 7, and 12 and are associated with loci that influence bitter perception in both humans and mice. Adler et al. also demonstrated that most or all of these putative bitter taste receptors (which are colocalized with gustducin) tend to be coexpressed in the same cells, suggesting that a given taste cell will respond to many bitter taste stimuli (cf. Caicedo \& Roper, 2001). Physiological analysis of selected Tas2rs, in an expression system (HEK-293 cells), by Chandrashekar et al. (2000) has provided highly compelling evidence that the proteins examined are in fact bitter taste receptors (Tas2r5, cycloheximide sensitive in mice, and TAS2R4, denatonium and PROP sensitive in humans).

The majority of psychophysical and physiological papers have focused on only a small subset of compounds. As was noted by Dahl et al. (1997), many neurophysiological studies have used only a single bitter compound- usually quinine. Alternatively, such studies have constructed analogs to study a single structure. Similarly, many psychophysical studies on bitterness have limited themselves to one or a few of the following compounds: quinine, caffeine, urea, PTC, and PROP (e.g., Fischer \& Griffin, 1963; Hall et al., 1975; Leach \& Noble, 1986; Schifferstein \& Frijters, 1991). Such limitation makes it difficult to find covariance in perceived intensities across compounds.

The present investigation employed some of the less frequently used classes of bitter-tasting compounds (e.g., amino acids such as L-phenylalanine and L-tryptophan, and nonamino acids such as denatonium benzoate and tetralone) and included at least one representative from most of the previously mentioned categories. The hope was to advance the understanding of bitterness perception by investigating individual differences in bitter perception through intercorrelations of bitterness sensitivities. For example, if all subjects insensitive to SOA are also insensitive to caffeine, and all subjects highly sensitive to SOA are similarly sensitive to caffeine, then one can predict that these two compounds have some bitter-taste transductive component in common, possibly a receptor. One can conservatively assume that bitter-tasting compounds that cluster together as a function of subjects' perceptual sensitivities share some common physiological mechanisms somewhere between the mouth and the brain.

\section{RATING}

\section{Method}

Subjects. Thirty-one paid volunteer subjects (10 men, 21 women; 18-35 years old) were recruited from the Monell Chemical Senses Center employees. They were instructed not to eat or drink anything other than water for the hour preceding each session. The Human Subjects Committee at the University of Pennsylvania approved all procedures. Two subjects failed to complete the study, 1 subject failed to comply with experimenter instructions, and 2 subjects failed to meet the consistency criterion in the ranking task (for further details, see Ranking section below); therefore, none of their data were included in the analyses. The following results derive from the data obtained from the remaining 26 subjects ( 8 men, 18 women; 18-34 years old).

Materials. Seven of the 10 compounds, and the concentrations at which they were used, were selected from compounds used by Guinard, Hong, Zoumas-Morse, Budwig, and Russell (1994). On average across individuals, these compounds were matched in intensity by Guinard et al. to $200 \mathrm{mM}$ sodium chloride by a paired-comparison, forced-choice, constant stimulus method by a panel of 17 trained subjects. These compounds, and their concentrations, were as follows: $1.19 \times 10^{-4} \mathrm{M}$ quinine hydrochloride, $5.70 \times 10^{-5} \mathrm{M}$ SOA, $9.20 \times 10^{-1} \mathrm{M}$ urea, $1.37 \times 10^{-4} \mathrm{M}$ tetralone, $5.02 \times 10^{-2} \mathrm{M} \mathrm{L}^{-}$ phenylalanine, $2.69 \times 10^{-2} \mathrm{M}$ L-tryptophan, and $1.09 \times 10^{-2} \mathrm{M}$ caffeine. Tetralone is a mixture of iso-alpha-acids. It is important to note that these were matches in average intensity across individuals and that these concentrations of compounds were not matched in intensity for any particular individual. Since the unrelated set of subjects in the present study would be highly unlikely to have the same average matched intensities, it was not necessary to find an exact match for the remaining three compounds. Guinard et al. did not use the remaining compounds (magnesium sulfate, denatonium benzoate, and (-)-epicatechin), so their concentration levels $(3.00 \times$ 
$10^{-1} \mathrm{M}, 4.99 \times 10^{-7} \mathrm{M}$, and $1.72 \times 10^{-2} \mathrm{M}$, respectively) were adjusted until they were each judged to fall into the same bitterness intensity range as the other seven compounds by 5 individuals. Although (-)-epicatechin is reported to be astringent at the level used in this study, none of the subjects found it to be so. Structures of all these compounds are available on request from the authors.

Procedure. Under red light and while wearing nose clips, the subjects were asked to rate the 10 solutions using the LMS scale (Green et al., 1996; Green, Shaffer, and Gilmore, 1993) for bitter intensity and for total intensity. The LMS is a pseudologarithmic scale that yields ratio-scale data and enables consideration of individual differences in suprathreshold ratings based on its labels. The scales were presented to the subject simultaneously, side by side, on a computer screen, with an adjustable pointer. The scale for bitter intensity was presented on the left, and that for total intensity was presented on the right. The subjects were instructed to use the same reference for the top of each scale, such that bitter intensity was a component of the total intensity and, therefore, either equal to or lower than the total intensity, but never higher than it. The subjects were instructed to taste the solution, rate it for bitter intensity, rinse twice, taste the solution again, and then rate it for total intensity. If they desired, the subjects were allowed to retaste the solution, and they were allowed to adjust their ratings until they were satisfied. The measurement of total intensity was required to prevent side tastes, if any were present, from being included in the bitterness rating.

Approximately $20 \mathrm{ml}$ of each solution were presented in a 1-oz (30-ml) plastic medicine cup (Premium Plastics, Inc., Chicago). During the 1-min interstimulus interval, the subjects rinsed a minimum of four times. The subjects received each solution twice per session in a random order, with all 10 solutions presented once before any were presented a second time. Every subject participated in three such sessions, the first of which was used as a training session and not included in subsequent analysis, resulting in four rating values for each compound from every subject.

PROP bitterness ratings and standardization with tone and weight ratings. To avoid introducing context effects into the ratings of the other bitter compounds arising from differences in PROP sensitivity (which are known to be substantial), the subjects assessed PROP in a single, separate session. In this session, they were asked to rate the bitter and total intensity of five levels of propylthiouracil $\left(5.50 \times 10^{-5} \mathrm{M}, 1.74 \times 10^{-4} \mathrm{M}, 5.50 \times 10^{-4} \mathrm{M}\right.$, $1.74 \times 10^{-3} \mathrm{M}$, and $\left.5.50 \times 10^{-3} \mathrm{M}\right)$ and water. The rating procedure, rinsing regimen, and interstimulus interval matched those of the rating sessions described above. In addition, the subjects were asked to rate the loudness of six tones (generated by a Maico Hearing Instruments tone generator, presented via headphones at $4000 \mathrm{~Hz}$ for $2 \mathrm{sec}$, at levels $0,20,35,50,65$, and $80 \mathrm{~dB}$ ) and the heaviness of six visually identical weights (opaque, sand-filled jars, at levels 225, 380, 558, 713, 870, and $999 \mathrm{~g}$ ). These ratings were made on a separate LMS scale that was described as a loudness scale for the former and a heaviness scale for the latter. All stimuli were rated two times, presented as two blocks, each in ascending order. The subjects first rated the intensity of PROP solutions, then tones, and, finally, weights.

For every subject, the intensity ratings were averaged across the three highest levels for each stimulus set. Significant correlations were found between PROP bitter intensity and loudness $(r=.45, p=$ $.022)$, PROP bitter intensity and heaviness $(r=.59, p=.001)$, and loudness and heaviness $(r=.55, p=.004)$. Since these variables should be unrelated, this indicated that the ratings were subject to bias and required standardization across subjects. Thus, to determine a correction factor, each subject's average intensity rating for loudness was divided by the grand mean for loudness across levels and subjects. The same was done with heaviness ratings. These two correction factors determined with loudness and heaviness were then averaged, and each subject's bitter and total intensity ratings for all 10 bitter compounds and all five levels of PROP were multiplied by his or her resulting average correction factor.
Normalization of LMS Ratings. After standardization, histograms of individual average bitter intensities and total intensities were plotted for all 10 compounds. In addition, the same was done with the middle concentration of PROP $\left(5.50 \times 10^{-4} \mathrm{M}\right)$, which, like the majority of the bitter compounds, was matched to $200 \mathrm{mM}$ $\mathrm{NaCl}$ by Guinard et al. (1994). As expected, the values followed a log-normal distribution (e.g., L-phenylalanine, as shown in Figure 1). By taking the log of the ratings, a normal distribution was approximated (e.g., L-phenylalanine, as shown in Figure 1). Therefore, the log was taken of all standardized LMS ratings before any statistical analyses were conducted. Before taking the log, all zero values were converted to .24 , the lowest possible value above zero that can be measured on the computerized LMS scale.

Standardization for cluster analysis. Since the compounds were not matched for intensity, it was necessary to centralize each compound's average intensity across subjects before performing a cluster analysis. Otherwise, the cluster analysis would have been driven by differences in intensity due to the particular concentration of each compound (which is not particularly interesting), rather than the underlying interrelationships between compounds (i.e., the covariation of compound ratings across subjects). Thus, an intensity correction factor was determined for each compound by dividing each compound's average rating across subjects by the grand mean (across compounds and subjects). Then, each subject's average rating (across replications) for a particular compound was multiplied by that compound's intensity correction factor; these were the values subsequently subjected to cluster analysis (using Statistica 5.0, single linkage joining, Ward's method, and squared Euclidean distances). In this manner, individual differences in bitterness ratings of compounds were maintained proportionately, but the average bitterness intensity across subjects for each compound was made the same.

\section{Rating Results}

The wide range of individual differences in the bitterness ratings can be seen in the top of Figure 2. Additionally, Pearson's product moment correlation coefficients were calculated for the intensities of all compounds. In order to reduce Type I errors, a Bonferroni correction was made to all $p$ values by multiplying each by 55 . No significant correlation was found between PROP and the 10 remaining compounds for either bitter or total intensities $(p>.05)$. This is especially striking since every other compound correlated significantly with at least one other compound, and the bitterness ratings of SOA correlated significantly with those of four other compounds (see Figure 2, bottom).

The results of principal components analysis (PCA, performed using Statistica 5.0 and presented unrotated) and cluster analysis (described above) for the bitter intensity data can be seen in Figure 3. The placement of the compounds in both analyses reveals relationships that are largely in accordance with the correlation matrices. In both analyses, at least two main groupings of compounds were revealed, neither of which contained PROP. One of these groups consists of phenylalanine, urea, tryptophan, and, more loosely, epicatechin, whereas the second group consists of the remaining compounds, excluding PROP. Analyses of the total intensities (not shown) were largely in agreement with those of the bitter intensities, although magnesium sulfate shifts away from the second group (tetralone, denatoniumbenzoate, SOA, caffeine, and quinine). This difference between analyses found for magnesium sulfate is most readily explained 

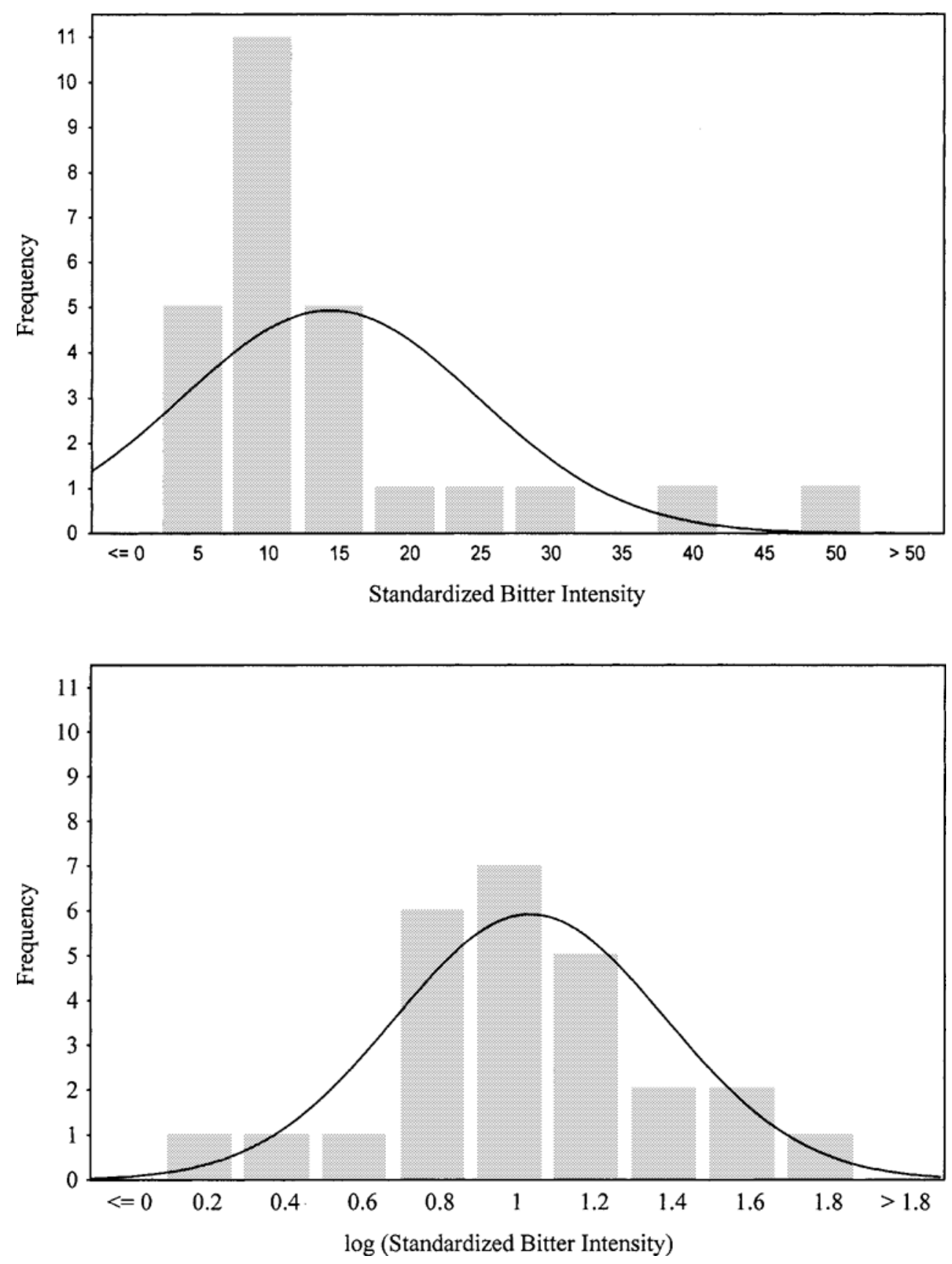

Figure 1. Histograms of L-phenylalanine standardized bitter intensity ratings before (top) and after (bottom) normalization.

by the fact that it has a relatively strong side taste (soursalty), which the other compounds lack.

\section{Discussion of Rating Results}

The PCAs and cluster analyses of the rating data indicate the presence of at least two groups of compounds, neither of which includes PROP (see Figure 3). Epicatechin is more loosely related to a clear ureaphenylalanine-tryptophan cluster in both the PCA and the cluster analyses, but the cluster analyses of both bitter and total intensity ratings show that it is in the same perceptual cluster. The cluster analyses of the bitter intensity ratings (see Figure 3, bottom) and total intensity ratings (not shown) indicates that tetralone and magnesium sulfate form their own subcluster, showing a closer relationship between each other than with the caffeine-SOAquinine-denatonium benzoate subcluster.

It is difficult to determine what underlying factor(s) is/are responsible for the clusters that appear in both the PCAs and the cluster analyses, but when one examines 


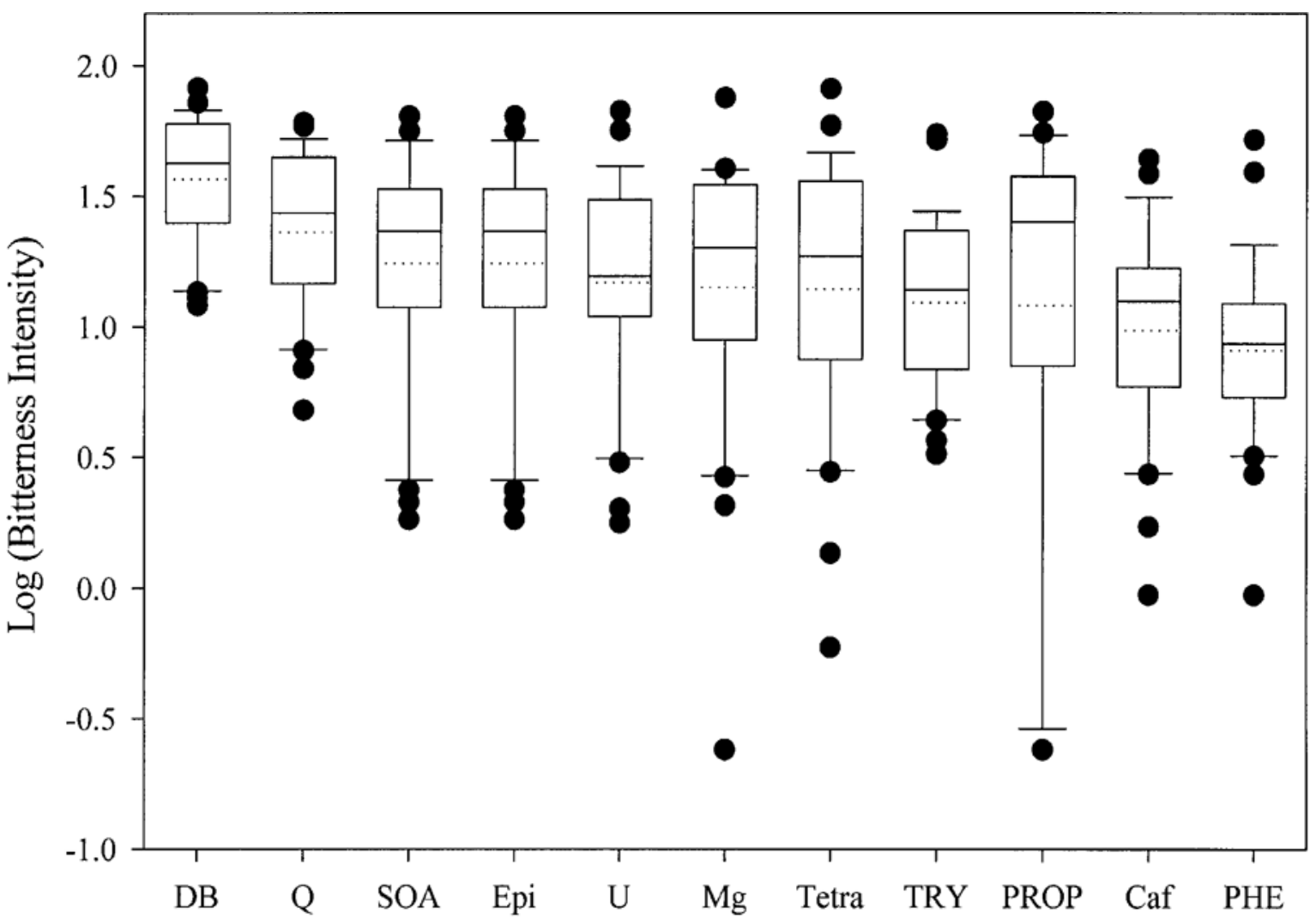

\begin{tabular}{|c|c|c|c|c|c|c|c|c|c|c|c|}
\hline & DB & $Q$ & SOA & Epi & $\mathbf{U}$ & Mg & Tetra & TRY & PROP & Caf & PHE \\
\hline DB & IIIIII & .66 & .71 & 26 & .33 & .48 & .67 & .29 & .3 & .63 & .12 \\
\hline $\mathbf{Q}$ & .63 & PMIMa & .69 & .26 & .64 & .53 & .6 & .5 & .28 & .67 & .48 \\
\hline SOA & .64 & .67 & POIII & .15 & .39 & 52 & .74 & .44 & .38 & .84 & .39 \\
\hline Epi & .26 & .23 & .12 & PIIs & .45 & .42 & .09 & .7 & .25 & .16 & .57 \\
\hline $\mathbf{U}$ & .28 & .49 & .32 & .53 & MIme & .37 & .26 & .65 & .45 & .47 & .62 \\
\hline $\mathrm{Mg}$ & .45 & .55 & .6 & .25 & .49 & 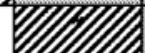 & .63 & .56 & .21 & 31 & .38 \\
\hline Tetra & .55 & .54 & .66 & -.01 & .17 & .7 & MOM. & .22 & .41 & .64 & .19 \\
\hline TRY & .21 & .4 & .37 & .71 & .6 & .45 & .1 & 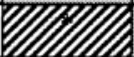 & .31 & .42 & .76 \\
\hline PROP & .24 & .26 & .39 & .24 & .43 & .34 & .52 & .33 & 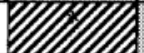 & 37 & .18 \\
\hline Caf & .53 & .63 & .81 & .09 & .46 & .52 & .61 & .31 & .37 & 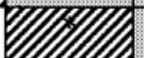 & .54 \\
\hline PHE & .03 & .42 & .3 & .47 & .65 & .35 & .06 & .68 & .16 & .52 & \\
\hline
\end{tabular}

Figure 2. Ratings. (Top) Box plot of bitter intensities. The bottom and top of each box designate the 25 th and 75 th percentiles, respectively, and the error bars designate the 10th and 90th. A solid line within the box represents the median, and a dashed line represents the mean. All outliers are shown as filled circles. (Bottom) Pearson's product moment correlation coefficients of ratings between compounds. Bitter intensity correlations are in unshaded cells; total intensity correlations are in shaded cells. Correlations in bold are significant at $p<.05$, post-Bonferroni correction. The following abbreviations were used: $D B=d e n a t o n i u m ~ b e n-$ zoate; $\mathbf{Q}=$ quinine hydrochloride; $\mathbf{S O A}=$ sucrose octaacetate; $\mathbf{E p i}=(-)$-epicatechin; $\mathrm{U}=$ urea; $\mathbf{M g}=$ magnesium sulfate; Tetra = tetralone; TRY = L-tryptophan; $\mathrm{PROP}=n$-propylthiouracil; Caf $=$ caffeine; and $\mathrm{PHE}=\mathrm{L}$-phenylalanine.

the chemical structure of these compounds, some similarities and differences can be seen. For example, phenylalanine, urea, and tryptophan all contain at least one primary amine, which none of the other compounds do. In contrast, tetralone, denatonium benzoate, SOA, caffeine, and quinine all contain at least one methyl group, which phenylalanine, urea, tryptophan, and epicatechin all lack. These trends can be quantified, and significant correla- 


\section{Bitter Intensity}
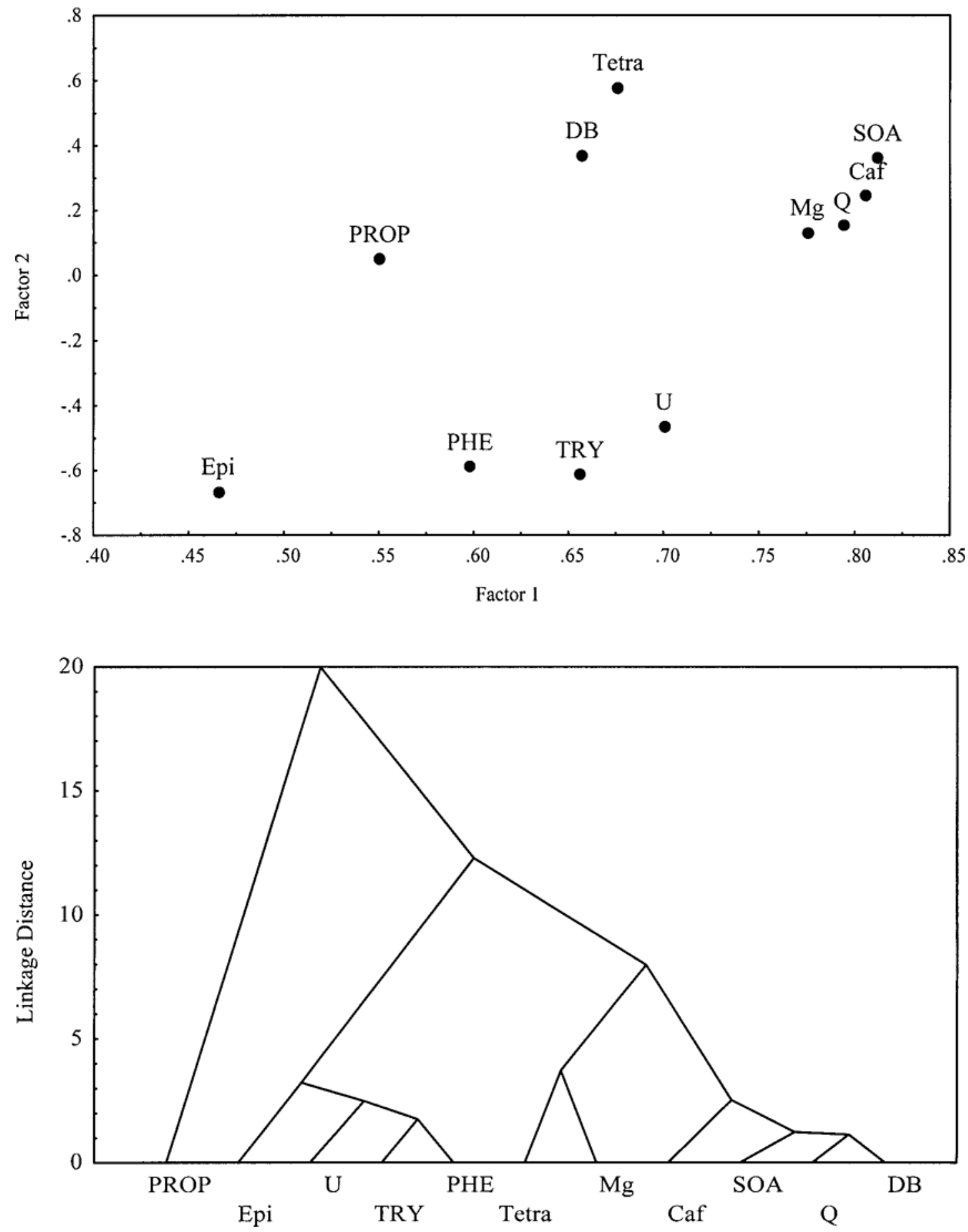

Figure 3. Bitter ratings. (Top) Principal components analysis of bitter intensities, presented unrotated. (Bottom) Cluster analysis of bitter intensities. The abbreviations used match those used in Figure 2.

tions between the number of these chemical moieties and PCA factor loadings can be found. In the PCA of the bitter ratings, the number of methyl groups a compound contains has a significant positive correlation with the compound's second factor loading $(r=.76, p=.007)$, whereas the number of primary amines a compound contains has a large negative correlation with the compound's second factor loading $(r=-.64, p=.033)$. Similar analyses of the total intensity ratings mirror these findings. 
Thus, there seems to be a separation between the compounds that contain methyl groups and those that contain primary amines, despite the fact that there are tremendous differences in the chemical classes of the compounds contained within the two main compound groups. Interestingly, epicatechin, which does not contain any primary amines or methyl groups, is the most weakly linked member of the cluster (see Figure 3). Additionally, magnesium sulfate, which also does not contain either chemical moiety, clearly stands in isolation within the PCA of the total intensity (not shown).

These observations of the chemical moiety differences among the compound clusters and the outliers do not fully account for the clustering of the compounds, but they could give insight into the types of transduction mechanisms involved in the perception of these bitter compounds, as well as how these transduction mechanisms are likely to differ. For example, the number of methyl groups will likely be positively correlated with compound lipophilicity (cf. Schiffman et al., 1994). Thus, this cluster may involve a mechanism dependent on lipophilic stimuli. No other physical parameters examined (including molecular weight, percent carbon, percent nitrogen, percent oxygen, hydrophobicity, and $\mathrm{pH}$ ) correlated significantly to the factor loadings.

\section{RANKING}

\section{Method}

Subjects. In order to directly compare rating data with ranking data, the 31 paid volunteer subjects who participated in the rating also participated in the ranking. As in the rating, 5 of these subjects were excluded; thus, the following results derive from the data obtained from the remaining 26 subjects ( 8 men, 18 women; $18-34$ years old).

Materials. With the exception of epicatechin (due to expense) and PROP (to avoid context effects), the nine compounds and concentrations used for the ratings were used for the rankings. PROP was not included, since it would necessarily introduce a priori contextual effects for the subjects who could not taste it and for those who were very sensitive to it.

Procedure. Under red light and while wearing nose clips, the subjects were asked to rank the nine solutions, from the weakest bitter to the strongest bitter. No ties were allowed. Approximately $30 \mathrm{ml}$ of each solution were presented in a 4-oz (118-ml) plastic souffle cup (Solo Cup Co., Urbana, IL). The subjects were allowed to taste and retaste the solutions as frequently as they desired at their own pace, but they were required to rinse twice between each solution. Cups were refilled as necessary. Each subject was asked to rank the solutions 15 times, but only once per session and no more than once per day.

R-index calculation and statistic. For each subject, an R-index matrix was created from the repeated rankings (O'Mahony, 1992). The R-index is a way of analyzing ranked data that allows one to see how far apart the stimuli are perceptually, and it was used to determine whether the subjects were ranking the compounds in a replicable way. For an example of this calculation, see the Appendix. It is a shortcut method derived from signal detection theory, and an Rindex value is equivalent to the probability that the judge can select one of two given stimuli when presented with both. R-index values can range from $100 \%$ (which indicates perfect discrimination) to $50 \%$ (which indicates chance). Intermediate values indicate less than perfect discrimination. The R-index matrix, built for each sub- ject, made it possible to compare each compound with every other compound; by using the table from Bi and O'Mahony (1995), it was determined whether the members of each stimulus pair were significantly different from one another. Over sessions, each of the 26 subjects generated his/her idiosyncratic, but highly reliable, rankorder, with slight fluctuations. Two of the original subjects (not included in the final 26 subjects) were highly inconsistent in their rankings, and over half the possible stimulus pairs failed to show a significant difference. Due to this lack of consistency, their data were not included in the analyses.

Paired comparisons. Once the R-index matrices had been calculated and the significance of each R-index value determined, each subject's matrix was examined. For each stimulus pair that did not show a significant difference, the subject was asked to do 20 forced-choice paired comparisons in a follow-up session to increase the likelihood that a significant ranked order could be established for each subject. The number of inconclusive stimulus pairs in a given subject's matrix varied from 3 to 15 , out of a possible 36 pairs. The subjects were asked to choose the more bitter compound of each pair. They were asked to sip from the first cup, then the second cup, and to repeat in reverse order, rinsing twice between each sip. This sipping regimen was selected since it was shown with pilot testing to yield the most consistent responses. The intertrial interval was $1 \mathrm{~min}$, and the subjects were instructed to rinse four times during this period.

If the subjects still failed to choose reliably one compound over the other in this paired comparison task, this could mean either (1) that the stimuli were completely indistinguishable or (2) that they were distinguishable but matched in bitterness intensity. To differentiate between these two possibilities, for any stimulus pair for which a given subject failed consistently to choose one compound as the most bitter, an additional session of 20 paired comparisons was completed by the subject. In this instance, however, before the stimulus pairs were assessed, the subject tasted both solutions several times and described any difference between them. The subject was then instructed to select the compound in each pair that was highest in this distinguishing characteristic. This technique is known as a warmed-up paired comparison (Thieme \& O'Mahony, 1990), and it allows one to use a paired comparison test even when the attribute that differentiates the two stimuli is difficult to articulate. An additional advantage of a warm-up is that it improves a subject's ability to distinguish between stimuli (O'Mahony, Thieme, \& Goldstein, 1988).

As mentioned above, all 26 subjects participated in at least three paired comparisons. Nine of the 26 subjects chose one compound as significantly more bitter than the other compound in all the paired comparisons required to completely clarify their significant ranked order. Seventeen subjects were still unable to reliably distinguish at least one of the stimulus pairs, and they were asked to distinguish the stimuli in subsequent warmed-up paired comparisons. The subjects managed to discriminate the stimuli under these circumstances for more than half of the stimulus pairs tested. This suggests that, although some of the compounds were very similar in bitterness intensity, they were perceptually distinguishable from one another. This was especially true of magnesium sulfate: Although it was one of the stimuli in one fifth of the warmed-up paired comparisons $(n=50)$, it was always discriminated under these circumstances (most likely due to its complex taste). Which stimuli were indistinguishable varied tremendously, and no other general trends were apparent. When the subjects did successfully distinguish between stimuli in a warmed-up paired comparison test (which occurred on 32 occasions), and in the rare instance when the difference in compounds was described as bitterness (only 2 of these 32 times), this information was used to further clarify the subject's significant ranked order.

Significant ranked order. From the results of the R-index calculations and the subsequent paired comparisons of bitterness, each 
subject's significant ranked order was determined. For any stimulus pairs that did not show a significant difference, or for any stimulus pair sets that resulted in nonrecursive orders (i.e., $\mathrm{A}>\mathrm{B}$, $\mathrm{B}>\mathrm{C}$, but $\mathrm{A}<\mathrm{C}$ ), the average rank was assigned to all involved stimuli. These rank values were used in all subsequent analyses.

Standardization for cluster analysis. As with the ratings, it was necessary to centralize each compound's average rank across subjects before performing a cluster analysis. Otherwise, as mentioned above, the cluster analysis would have been driven by differences in intensity due to the particular concentration of each compound, rather than the covariation of compound rankings across subjects. As with the ratings, an intensity correction factor was determined for each compound by dividing each compound's average rank across subjects by the grand mean (across ranks and subjects). Then, each subject's rank for a particular compound was multiplied by that compound's intensity correction factor; these were the values subjected to cluster analysis (using, as above, Statistica 5.0, single linkage joining, Ward's method, and squared Euclidean distances).

\section{Ranking Results}

The wide spread of individual differences in the bitterness rankings can be seen in the top of Figure 4. Additionally, Spearman's rho correlation coefficient was calculated for the bitter ranks of all compounds. In order to reduce Type I errors, a Bonferroni correction was made to all $p$ values by multiplying each by 36 . Several significant correlations were found (see Figure 4, bottom), but, unlike with bitterness intensities, over half of the significant correlations were negative (see explanation for this apparent discrepancy below). Caffeine was negatively correlated to urea and tryptophan and was positively correlated to SOA and tetralone. In addition, tetralone was negatively correlated to tryptophan and phenylalanine, which were in turn positively correlated to one another.

A PCA (performed using Statistica 5.0 and presented unrotated) and cluster analysis of the bitterness rankings can be seen in Figure 5, and the compound groupings are largely in accordance with the correlation matrix and similar to that found with the ratings. As before, two broad groupings of compounds were revealed in both analyses, the tightest of which consisted of phenylalanine, tryptophan, and urea, whereas a looser group consisted of denatonium, SOA, caffeine, and tetralone. Again, the trend to separate the compounds with primary amines from those with methyl groups was observed. The number of methyl groups a compound contains has a significant positive correlation with the compound's first factor loading ( $r=.84, p=.005)$, and the number of primary amines a compound contains has a significant negative correlation with the compound's first factor loading ( $r=$ $-.78, p=.013)$.

\section{Discussion of Ranking Results}

Several differences can be seen between the Pearson's product moment correlation coefficient matrix of the bitterness ratings and the Spearman's rho correlation coefficient matrix for the ranks of the same compounds (see
Figures 2, 4, and 6). Very different correlations are significant between the two sets of data, although three compound pairs showed significant correlations in both data sets: caffeine/SOA, caffeine/tetralone, and phenylalanine/ tryptophan. The majority of significant correlations in the ranking data were negative, whereas no significant negative correlations were found with the bitterness ratings. However, it is important to note that each significant negative correlation found for the ranking data was a nonsignificant correlation for the ratings.

The reason for the apparent discrepancies between the correlations based on ratings and those based on rankings can be attributed to differences in overall bitterness sensitivities among subjects (see PROP Status section below). Some subjects consistently gave lower bitterness ratings to all compounds, whereas others consistently gave high bitterness ratings to all compounds. In other words, the apparent difference in outcomes between procedures was due to the fact that the ranking methodology is insensitive to absolute differences between subjects in perceived intensities, allowing negative correlations to be revealed. With rating procedures, some subjects consistently give higher ratings and others give lower ratings to all bitter compounds, leading to positive correlations across subjects. As a consequence, one would expect negative correlations in the ranking data to become weakly positive correlations in the rating data, which is what happened in the present experiment.

As was seen with the rating data, the PCA and cluster analysis of the ranking data reveal at least two groups of compounds (see Figure 5). However, in the ranking analyses, neither group contains magnesium sulfate. Phenylalanine, tryptophan, and urea are tightly clustered in all rating and ranking analyses, indicating a very strong association of these three compounds. Similar to the rating analyses, the second general cluster in both the ranking cluster analysis and the PCA contains caffeine, tetralone, quinine, SOA, and denatonium benzoate. All significant negative Spearman's rho correlations are found between members of different clusters; no significant negative correlations are found between the compounds within a cluster.

Why magnesium sulfate stands as an outlier in the PCA and cluster analysis of bitter rankings (see Figure 5), but not those of the bitter ratings (see Figure 3), could be accounted for by the possibility of "dumping" effects occurring in the ranking task (Frank, Van der Klaauw, \& Schifferstein, 1993; Lawless \& Clark, 1992). In the rating task, subjects are given the opportunity to simultaneously rate compounds for both bitter and total intensities. This means that if a compound has a prominent taste other than bitterness, subjects have the opportunity to express this on the total intensity scale. Magnesium sulfate does have a prominent taste other than bitterness (sourness-saltiness), as evidenced by the difference between the mean bitter and total intensity ratings of 22.5 and 31.5; in the total intensity PCA, it failed to cluster tightly with any other compound. In the ranking task, 


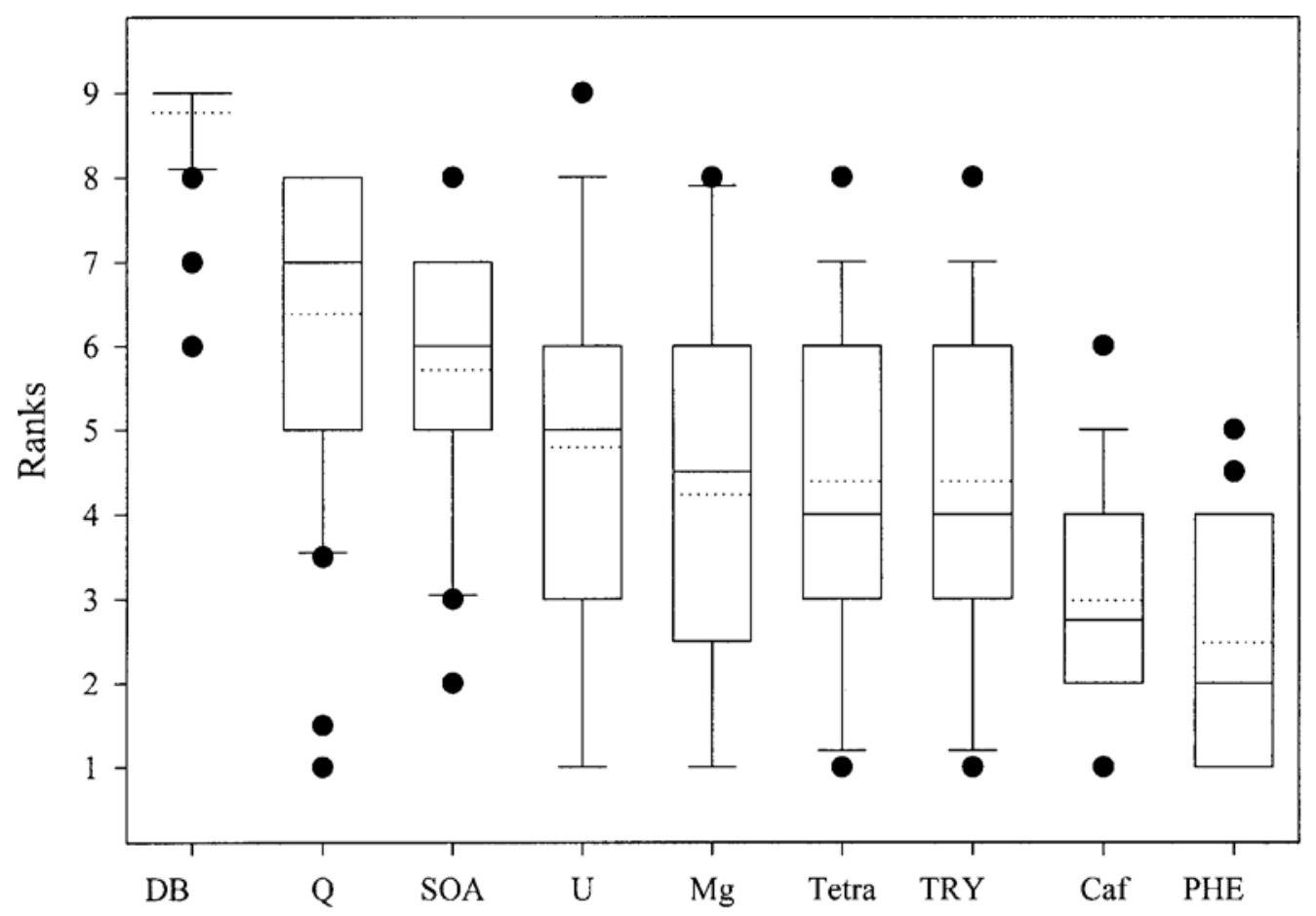

\begin{tabular}{|c|c|c|c|c|c|c|c|c|c|}
\hline & DB & Q & SOA & U & Mg & Tetra & TRY & Caf & PHE \\
\hline DB & $*$ & & & & & & & & \\
\hline Q & .38 & $*$ & & & & & & & \\
\hline SOA & .50 & -.09 & $*$ & & & & & & \\
\hline U & -.50 & -.22 & -.47 & $*$ & & & & & \\
\hline Mg & -.47 & -.51 & -.37 & .06 & $*$ & & & & \\
\hline Tetra & .26 & -.19 & .33 & -.54 & .01 & $*$ & & & \\
\hline TRY & -.44 & -.05 & -.53 & .46 & .01 & -.70 & $*$ & & \\
\hline Caf & .51 & .04 & .68 & -.61 & -.34 &. $\mathbf{6 2}$ & -.63 & $*$ & \\
\hline PHE & -.47 & -.03 & -.39 & .46 & -.09 & -.60 & .58 & -.57 & $*$ \\
\hline
\end{tabular}

Figure 4. Rankings. (Top) Box plot of bitter rankings (same as Figure 2, top). There is no visible box or median for denatonium benzoate because the 90th, 75 th, 50 th, and 25 th percentiles were identical. (Bottom) Spearman's rho correlation coefficients of bitter ranking between compounds. Correlations in bold are significant at $p<.05$, post-Bonferroni correction. The abbreviations used match those used in Figure 2 .

since the compounds were ranked only for bitterness and never for total intensity, the subjects did not have a way to express their perception of the prominent side taste many experience when tasting magnesium sulfate. Thus, when ranking magnesium sulfate, the subjects' judgments may have been influenced, thus resulting in the discrepancy found between the PCAs. These kinds of dumping effects have been found in many instances in which the assessment task does not allow subjects to respond to a salient perceptual feature (Frank et al., 1993; Lawless \& Clark, 1992).
As discussed in the previous section and as was found with the rating data, a separation between the compounds that contain methyl groups and those that contain primary amines was found along the first factor in the PCA, despite the tremendous differences in the chemical classes of the compounds contained within the two main perceptual clusters. Magnesium sulfate, which does not contain any primary amines or methyl groups, can be viewed as an isolated compound. Quinine, which contains one methyl group, is also more isolated from the other compounds (see Figure 5, top). As with the ratings, no other physical 

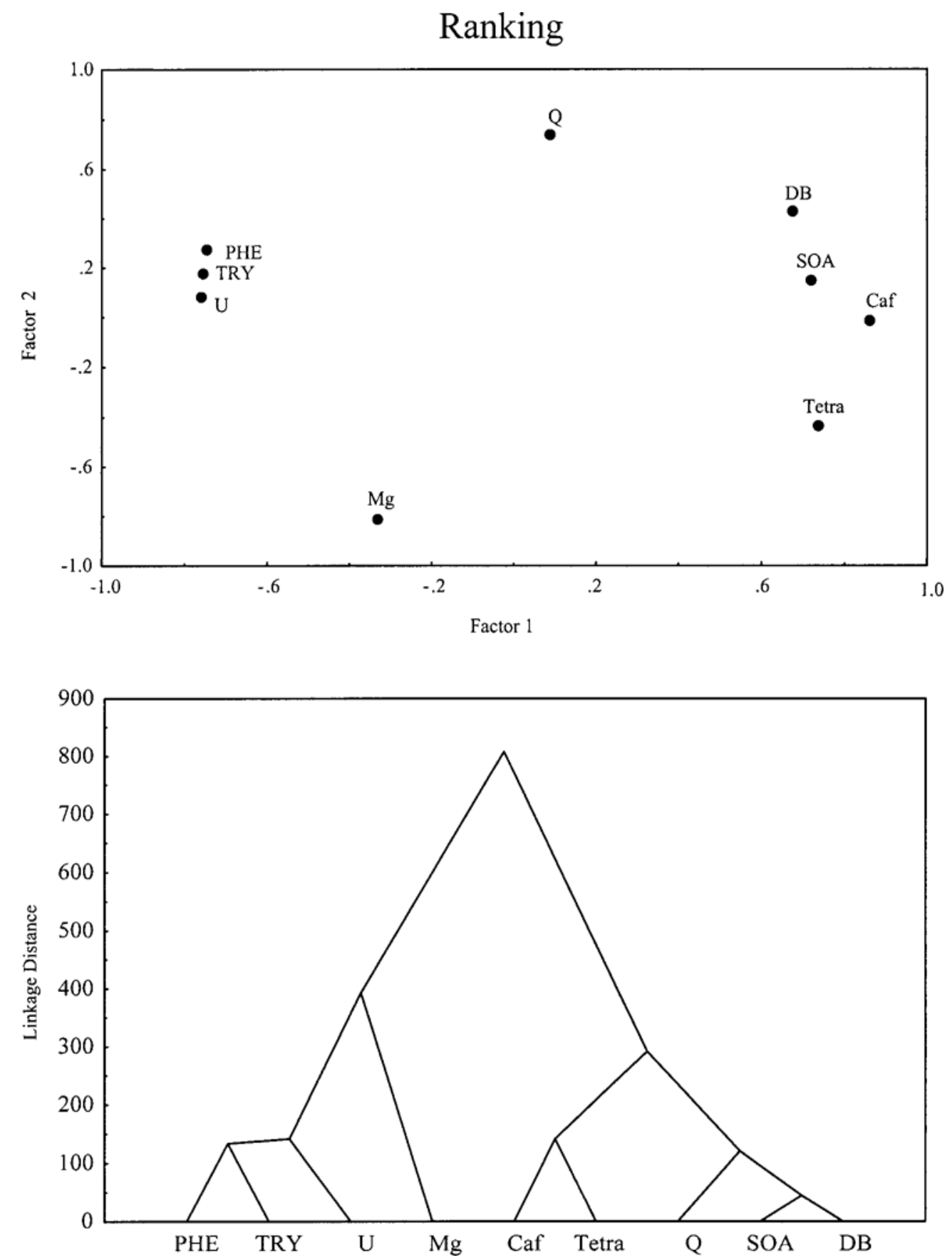

Figure 5. Rankings. (Top) Principal components analysis of bitter rankings, presented unrotated. (Bottom) Cluster analysis of bitter rankings. The abbreviations used match those used in Figure 2.

parameters examined (including molecular weight, percent carbon, percent nitrogen, percent oxygen, hydrophobicity, and $\mathrm{pH}$ ) correlated significantly to the factor loadings. It is clear that the chemical moiety differences between the compound clusters and the outliers do not perfectly account for the perceptual clustering of the compounds, but they are still suggestive of the types of transduction mechanisms involved in the perception of these bitter compounds and how these transduction mechanisms are likely to differ.

When the totality of both the rating and the ranking results is considered, the data seem to indicate an especially close association of phenylalanine, tryptophan, and urea, as well as an association between caffeine, SOA, quinine, and denatonium benzoate, which are even less closely associated with tetralone. The data also indicate that PROP 


\section{Bitter Intensity}
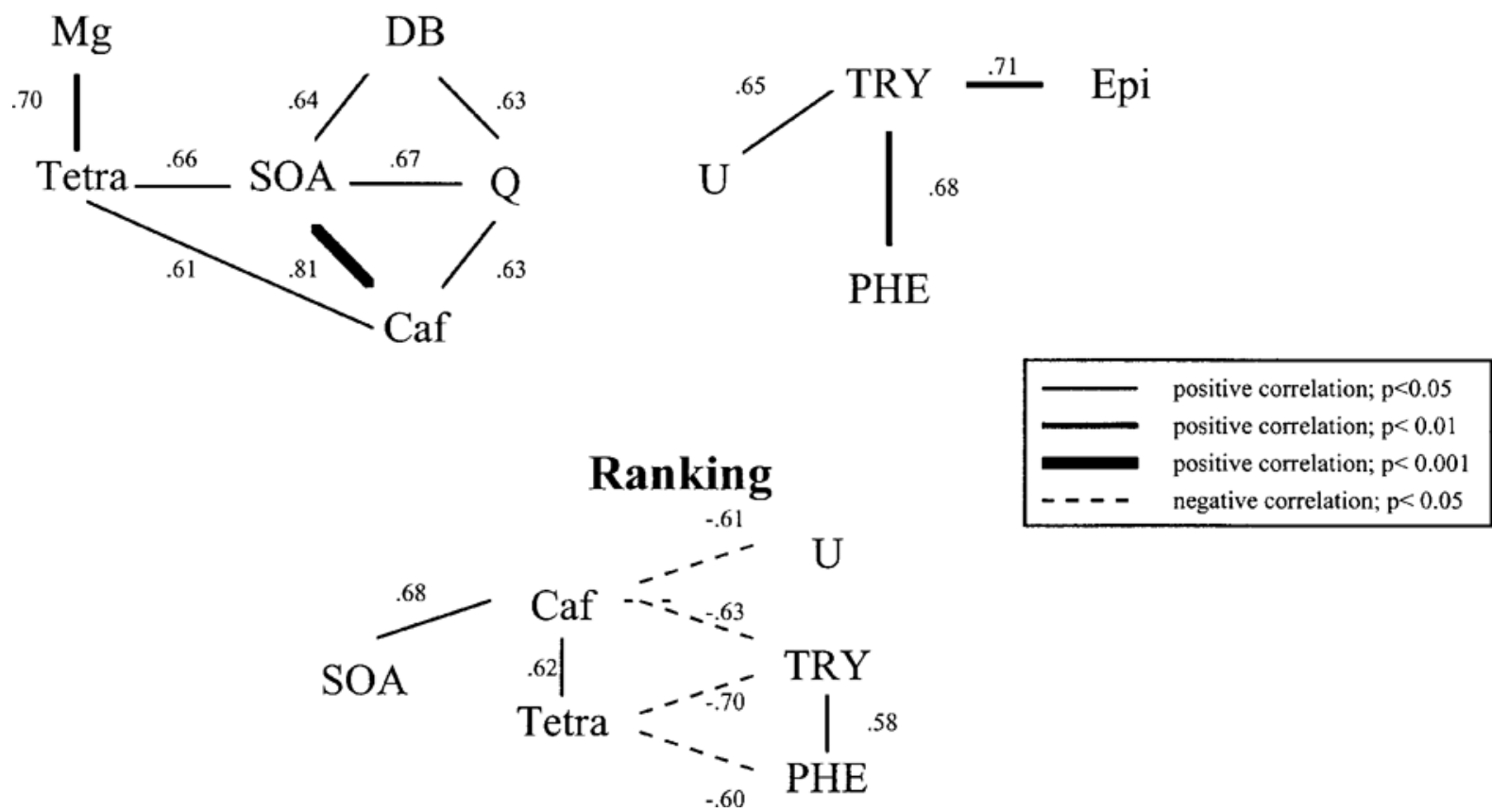

Figure 6. Summary of significant correlations across assessments. Significant correlations between compounds are indicated by lines (paired with corresponding correlation coefficients) between the appropriate compounds. Thickness of lines indicates significance, post-Bonferroni correction. Dashed lines indicate negative correlations. (Top) Significant correlations of bitter intensities. (Bottom) Significant correlations of bitterness rankings. The abbreviations used match those used in Figure 2.

is perceptually dissimilar to all the other compounds assessed. Other relationships (i.e., that of epicatechin and magnesium sulfate) seem more tentative and dependent on the experimental task.

\section{PROP STATUS}

PROP status was established by each subject's average bitterness rating of the highest level of PROP $(5.50 \times$ $10^{-3} \mathrm{M}$ PROP) given in the standardization session (see the Method section). The groupings were based on arbitrary criteria to select the extreme ends of the PROP distributions. The subjects who gave the four highest ratings (above 82, poststandardization, on the LMS scale) were classified as super tasters, whereas the subjects who gave the four lowest ratings (below 11, poststandardization, on the LMS scale) were classified as nontasters. The 18 remaining subjects were classified as (medium) tasters. The standardization process, in part, caused the unusually high ratings on the LMS by super tasters.

Using Statistica 5.0, a repeated-measures analysis of variance (ANOVA) was performed with PROP status as an independent variable on the bitter and the total intensity ratings, and a Kruskal-Wallis ANOVA by ranks was performed on the rankings. As expected, for $5.50 \times 10^{-4} \mathrm{M}$ PROP (intensity matched by the Guinard et al., 1994, panel to $200 \mathrm{mM} \mathrm{NaCl}$ ), significant differences in bitterness were found between the status groups $(p<.05$, supporting the status classification of the subjects) but were not found for loudness or heaviness $(p>.05$, indicating that the correction factors were successful at standardizing the data). The bitterness ratings (see Figure 7, top) and total intensity ratings (not shown) of the different PROP status groups were both significantly different $(p<.01)$, although, due to the low power associated with having only four subjects in two of the groups, a Scheffé's test failed to reveal significant differences between the specific status groups for each particular compound. In contrast, no significant difference in rankings was found between the PROP status groups (see Figure 7, bottom).

The finding that subjects who are insensitive to PROP give lower ratings to bitter compounds than do subjects more sensitive to PROP has been found by other researchers as well (e.g., Lawless, 1979; Schiffman et al., 1994). Although these results initially may seem to contradict the lack of PROP correlations with other compounds reported above, the apparent discrepancy is due to the fact that only a very few subjects were shown to rate all compounds consistently lower than other subjects. In the correlational analyses, the impact of these few was sufficient to create a weakly positive, but nonsignificant, correlation (see Figure 2, bottom). Only if the subjects 

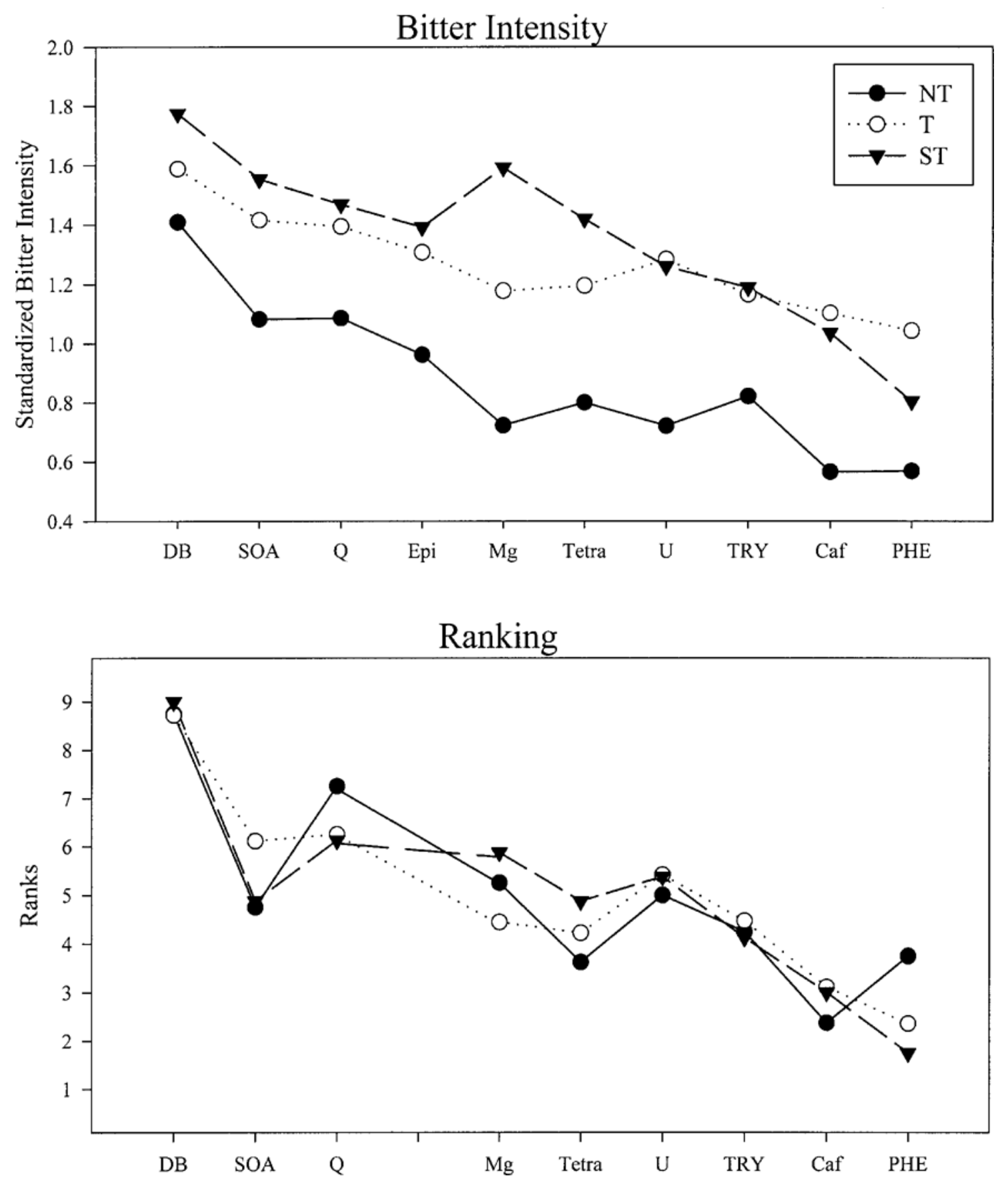

Figure 7. Influence of PROP status. Average across subjects in each PROP status group for bitter intensities (top) and bitter rankings (bottom) of each compound. Nontasters (NT) are indicated by closed circles connected by solid lines, medium tasters (T) by open circles connected by dotted lines, and super tasters (ST) by closed triangles connected by dashed lines. The abbreviations used match those used in Figure 2. Note: Epi was not used in ranking.

are categorized such that these few insensitive subjects are separated from the others can the influence of PROP status be demonstrated.

There is, however, no significant difference between these subjects in the rankings given to these compounds. This seems to indicate that the subjects of each group differ only quantitatively, not qualitatively. In other words, nontasters have lower system gains for bitterness than do medium and super tasters. This is perhaps related to the number of taste buds and/or taste pores, as has been suggested by Bartoshuk, Duffy, and Miller (1994), although increased or decreased numbers of taste buds might be expected to affect all taste qualities, not just bitterness. These results indicate that general sensitivity to bitter 
compounds across the board is one factor that is independent of the issue of individual differences in responses to different compounds due to variation in transduction mechanisms. In group comparisons, the general sensitivity effects can confound the measurement of sensitivities to individual compounds.

\section{GENERAL DISCUSSION}

The guiding hypothesis for this work is that bittertasting compounds that cluster together as a function of individual differences in subjects' perceptual sensitivities are likely to share a common physiological mechanism somewhere between the mouth and the brain, most probably in the receptor cell transduction sequence. In order to maximize the chemical diversity of stimuli, at least one representative from several of the less frequently used bitter chemical categories was included. The findings revealed clear evidence for correlations among bitter compounds as a function of individual differences in subject sensitivities (illustrated in Figures 2, 4, and 6) and reliable groupings of compounds into subclasses (illustrated in Figures 3 and 5).

On the basis of this study, there appear to be four major clusters of bitter compounds: (1) denatonium benzoate, tetralone, caffeine, SOA, and quinine; (2) urea, tryptophan, phenylalanine, and epicatechin; (3) magnesium sulfate; and (4) PROP. All the compounds in Group 1 contain at least one methyl group, whereas three of the four compounds in Group 2 contain one or more primary amines. Within these first two groups, there also appear to be subclusters of compounds that are more closely affiliated to one another, although these subclusters vary from one analysis to another.

In general, these observations have been supported by a variety of human psychophysical studies, including those involving bitterness ratings, threshold measurements, cross-adaptation studies, and suppression of bitterness by sodium. These groupings have also been supported by mouse behavioral genetics focusing on the Soa locus.

\section{Human Psychophysical Studies}

Cross-adaptation and individual sensitivities. Yokomukai et al. (1993), Lawless (1979), and McBurney et al. (1972) interpreted their sensitivity and cross-adaptation data to suggest at least three separate, although perhaps overlapping, bitter transduction sequences. The three posited were one particularly sensitive to PTC and related compounds, one particularly sensitive to the bitterness of quinine, caffeine, and SOA, and one particularly sensitive to urea, magnesium sulfate, and creatinine. These findings are largely in agreement with those of the present study. Both the present study and the studies of Yokomukai et al. and Lawless found PROP/PTC sensitivity to be independent of other bitter compounds, which resonates with the findings in mice (Whitney \& Harder, 1994). Yokomukai et al. and McBurney et al. also suggest a tight relationship among quinine, caffeine, and SOA, which was found in the rating assessments of the present investigation. The present ranking data, however, did not cluster quinine so tightly with caffeine and SOA, which is more in agreement with the findings in mice (Boughter \& Whitney, 1998). In further agreement, Yokomukai et al. (1993) found that urea-sensitive subjects rated caffeine as less bitter than did quinine-sensitive subjects, indicating a negative correlation between urea and caffeine, as was found with the ranking data of the present investigation. In agreement with the present clustering of urea with amino acids, Lawless found creatinine (an amino acid-like compound containing both a carboxylic group and a guanidinium group) to mutually cross-adapt urea.

The largest disagreement between the findings of the present study and those of Yokomukai et al. (1993) and McBurney et al. (1972) is the suggested grouping of urea and magnesium sulfate. In the present investigation, urea was found to be in a group with phenylalanine and tryptophan, separate from the other compounds, whereas magnesium sulfate did not fall into this cluster. Magnesium sulfate clusters with urea, phenylalanine, and tryptophan in the cluster analysis of the ranking data (see Figure 5), but this is a loose linkage, and it is actually more of an outlier in this analysis. In the cluster analyses of the rating data, magnesium sulfate clusters most closely to tetralone within the broad cluster of quinine/caffeine/ SOA/denatonium benzoate/tetralone/magnesium sulfate. Perhaps phenylalanine and tryptophan are more similar to urea than to magnesium sulfate, and their presence in the stimuli set caused magnesium sulfate (which has a complex bitter-sour-salty taste) to seem more perceptually dissimilar from urea.

Suppression by sodium. Other evidence exists that indicates perceptual differences in the transduction of urea and magnesium sulfate. Breslin and Beauchamp (1995) examined the effect of sodium on bitterness suppression and found differential suppression of bitterness across intensity-matched concentrations of urea, quinine $\mathrm{HCl}$, magnesium sulfate, and caffeine. Both caffeine and quinine were moderately suppressed in bitterness; urea was almost completely suppressed, whereas the bitterness of magnesium sulfate was largely unaffected (Breslin \& Beauchamp, 1995). Thus, the effectiveness of suppression in bitterness by sodium across compounds separates the compounds into three classes (urea; quinine and caffeine; and magnesium sulfate), matching the findings of the present investigation.

Correlations among PROP/PTC and other bitter compounds. The best documented individual differences in human perception of bitter compounds have been found for PROP and PTC (e.g., Bartoshuk et al., 1996; Fischer \& Griffin, 1963; Hall et al., 1975; Kemp \& Birch, 1992; Thorngate, 1997; Yokomukai et al., 1993). In such studies, subjects are typically divided into two (nontaster and taster) or three (nontaster, taster, and super taster) groups (Reed, Bartoshuk, Duffy, Marino, \& Price, 1995). In agreement with the findings of this study, how strongly 
one perceives a particular concentration of PROP has not proven to be a reliable predictor of how strongly one perceives particular concentrations of other bitter substances (unless those who are highly insensitive are examined separately). In fact, subjects who are highly sensitive to one bitter compound can be quite insensitive to another (Yokomukai et al., 1993).

Several studies have investigated the relationship between PROP (or PTC) and quinine. Although some studies have found a significant positive relationship between PROP or PTC sensitivity and quinine sensitivity (Gent \& Bartoshuk, 1983; Jefferson \& Erdman, 1970; Lawless, 1979; Leach \& Noble, 1986), several other studies have failed to find such a relationship either for suprathreshold ratings of quinine (Bartoshuk, 1979; Bartoshuk, Rifkin, Marks, \& Hooper, 1988; Frank \& Korchmar, 1985; Mela, 1989; Schifferstein \& Frijters, 1991; Yokomukai et al., 1993) or for quinine thresholds (Fischer \& Griffin, 1963; Hall et al., 1975; Lawless, 1979; Schiffman et al., 1994).

Fewer studies have investigated the relationship between PROP/PTC and caffeine, but these studies have shown no more agreement. Hall et al. (1975) found that "sensitivity to the taste of PTC predicts sensitivity to caffeine," using both threshold measurements and magnitude estimations of suprathreshold concentrations of caffeine, but other studies failed to find a significant difference between tasters and nontasters in the ratings of suprathreshold concentrations of caffeine (Leach \& Noble, 1986; Mela, 1989) or in caffeine thresholds (Schiffman et al., 1994).

Similarly, studies have failed to find a clear relationship between urea and PROP/PTC (Hall et al., 1975; Mela, 1989; Yokomukai et al., 1993), at either threshold levels (Hall et al., 1975; Schiffman et al., 1994) or suprathreshold concentrations (Mela, 1989; Yokomukai et al., 1993). Following this trend, Mela (1989) failed to find a significant difference between PROP tasters and nontasters in the suprathreshold concentrations of SOA and denatonium benzoate, and both Schiffman et al. (1994) and Boughter and Whitney (1993) failed to find a significant difference between PROP taster groups in the thresholds of SOA. Schiffman et al. also failed to find a significant difference between PROP taster groups for the thresholds of denatonium benzoate and magnesium sulfate. In a later study by Mela (1990), PROP tasters rated isohumulone significantly more bitter than did nontasters when isohumulone was presented in water, but not when it was presented in beer. Lawless (1979) failed to find any relationship between sensitivity to PTC and creatine or creatinine at threshold or suprathreshold levels.

These findings, together with the findings of the present study, indicate that the relationship between the perception of PTC/PROP and the perception of other bitter compounds is not a simple one. Some of the contradictions across studies could have arisen from the percentage of PROP/PTC-insensitive subjects varying from one study to another; studies with a higher percentage of nontasters would be more likely to find a significant relation- ship between PROP/PTC and other compounds than with those with a lower percentage. In addition, as demonstrated in the present study, whether statistical analyses considered nontasters as a separate category would also impact on whether or not PROP/PTC status appeared to influence sensitivity to other bitter compounds. Finally, the use of scales with ceilings and the use of designated intensity standards may tend to minimize differences across subjects (Bartoshuk et al., 1999).

Correlations among bitter compounds in mice. Behavioral genetic evidence in mice has identified at least three genetic loci that influence differences in response to bitter compounds (Whitney \& Harder, 1994). These findings show a very strong parallel to the present human data, as described below. A single locus (Soa) accounts for virtually all of the variation in response to SOA (Capeless, Whitney, \& Azen, 1992; Harder, Gannon, \& Whitney, 1996; Harder, Whitney, Frye, Smith, \& Rashotte, 1984; Whitney \& Harder, 1994), whereas responses to quinine sulfate are influenced by at least two unlinked loci, one of which is Soa (Boughter \& Whitney, 1998; Whitney \& Harder, 1994). A third locus seems to be involved in responses to PTC (Whitney \& Harder, 1994), but unlike in humans, sensitivity to PROP and PTC are uncorrelated in mice (Harder, Boughter, \& Whitney, 1996; Harder \& Whitney, 1998).

Similar to the findings of the present study, SOA and quinine aversions in mice were substantially correlated, whereas aversion to either failed to correlate with PTC aversion (Whitney \& Harder, 1994). Isohumulone was clearly influenced by the Soa locus (Whitney \& Harder, 1994), which parallels the loose clustering of tetralone with SOA found in the present investigation. Sensitivity to denatonium benzoate also seems to be influenced by the Soa locus (Boughter \& Whitney, 1998; Whitney \& Harder, 1994), whereas sensitivity to L-phenylalanine does not (Harder, Gannon, \& Whitney, 1996), which also agrees with the present findings. In contrast to the present findings, however, caffeine sensitivity was not influenced by the Soa locus (Boughter \& Whitney, 1998), whereas sensitivity to PROP was (Harder \& Whitney, 1998). The recent work of Adler et al. (2000) and Matsunami et al. (2000) on bitter receptor genes suggests there may be as many as 80 bitter taste receptor genes that will require behavioral and physiological characterization in mice and humans.

The global insensitivity of the PROP nontasters to bitter compounds, found in the present study and noted also by Lawless (1979), could be explained by a reduced number of taste receptors, relative to that of PROP medium and super tasters. Miller and Whitney (1989) have found that SOA-taster mice (those with lower aversion thresholds) have more vallate taste buds than do SOA-nontasters (those with higher aversion thresholds), whereas Shingai and Beidler (1985) found that relative taste responses from SOA in the glossopharyngeal nerves of SOA-taster mice are larger in magnitude than are responses to the same stimuli from SOA-nontasters. Similarly, Bartoshuk 
et al. (1994) found a link between the perceived bitterness of PROP and the density of taste receptors on the anterior human tongue. Since stimulation of an increasing number of taste buds in a population of fungiform papillae is known to give rise to progressively greater perceived taste intensities (Arvidson \& Friberg, 1980; Smith, 1971), both mouse and human data suggest that the number of taste buds contributes to the absolute differences in taste sensitivities and preference behaviors. But, spatial summation should also result in the mice and the humans with greater numbers of taste buds experiencing greater intensities of salts, acids, sweets, and all stimuli. One should note too that the range of fungiform papillae density of the medium taster group completely encompasses that of both nontasters and super tasters (Bartoshuk et al., 1994). Thus, although the correlation of sensitivity and number of buds/papillae is certainly striking, it simply does not seem to tell the whole story; transductive differences likely also account for differences across groups.

\section{Conclusions}

Sensitivity to any bitter compound may be affected in part by the type of transduction sequence involved for that compound. Thus, within a subject, two compounds that share transduction components should be similarly affected by polymorphic changes in relevant transduction proteins. When measured across many subjects, significant correlations of sensitivities to certain compounds strongly suggest that those compounds share common transduction components. Bitterness, therefore, appears to be transduced in humans via several heterogeneous interactions; among the 11 compounds employed in the present study, groupings suggest four different transduction mechanisms, a high ratio of bitter compounds to transduction mechanisms. These individual differences in responses to different bitter compounds (which suggest variation in transduction mechanisms) can be recognized only when differences in global sensitivity to bitter compounds are not allowed to confound the former. Given the vast number of bitter-tasting compounds and their structural diversity, it is perhaps not surprising that Adler et al. (2000) and Matsunami et al. (2000) have suggested 80 (and possibly more) bitter taste receptors in humans and mice. Their reports support the relatively large number of independent groupings found with only 11 stimuli in the present study.

It is important to point out that the clusterings in this study relate only to sensitivity to bitter compounds. They do not address issues of bitterness quality or the possibility of multiple bitter subqualities. For example, all transduction interactions may involve the same bitter-sensitive taste receptor cells as suggested by Adler et al., 2000) or converge onto the same primary neuron, in which case all compounds, regardless of their cluster identity or transductive mechanisms, would still give rise to the same quality of bitter taste.

\section{REFERENCES}

Adler, E., Hoon, M. A., Mueller, K. L., Chandrashekar, J., RybA, N. J. P., \& ZuKer, C. S. (2000). A novel family of mammalian taste receptors. Cell, 100, 693-702.

Arvidson, K., \& Friberg, U. (1980). Human taste: Response and taste bud number in fungiform papillae. Science, 209, 807-808.

Barnicot, N. A., Harris, H., \& Kalmus, H. (1951). Taste thresholds of further eighteen compounds and their correlation with P.T.C. thresholds. Annals of Eugenics, 16, 119-128.

BARToshuk, L. M. (1979). Bitter taste of saccharin related to the genetic ability to taste the bitter substance 6- $n$-propylthiouracil. Science, 205, 934-935.

Bartoshuk, L. M., Cunningham, K. E., Dabrila, G. M., Duffy, V. B., Etter, L., Fast, K. R., Lucchina, L. A., Prutkin, J. M., \& Snyder, D. J. (1999). From sweets to hot peppers: Genetic variation in taste, oral pain, and oral touch. In G. A. Bell \& A. J. Watson (Eds.), Tastes \& aromas (pp. 12-22). Sydney: University of New South Wales Press.

Bartoshuk, L. M., Duffy, V. B., \& Miller, I. J. (1994). PTC/PROP tasting: Anatomy, psychophysics, and sex effects. Physiology \& Behavior, 56, 1165-1171.

Bartoshuk, L. M., Duffy, V. B., Reed, D., \& Williams, A. (1996). Supertasting, earaches and head injury: Genetics and pathology alter our taste worlds. Neuroscience \& Biobehavioral Review, 20, 79-87.

Bartoshuk, L. M., Rifkin, B., Marks, L. E., \& Hooper, J. E. (1988). Bitterness of $\mathrm{KCl}$ and benzoate: Related to genetic status for sensitivity to PTC/PROP. Chemical Senses, 13, 517-528.

BelitZ, H.-D., \& WIESER, H. (1985). Bitter compounds: Occurrence and structure-activity relationships. Food Reviews International, 1, 271-354.

Bi, J., \& O'MAHony, M. (1995). Table for testing the significance of the R-index. Journal of Sensory Studies, 10, 341-347.

Boughter, J. D., JR., \& Whitney, G. (1993). Human taste thresholds for sucrose octaacetate. Chemical Senses, 18, 445-448.

Boughter, J. D., JR., \& Whitney, G. (1998). Behavioral specificity of the bitter taste gene Soa. Physiology \& Behavior, 63, 101-108.

BRAND, J. G. (1997). Biophysics of taste. In G. K. Beauchamp \& L. Bartoshuk (Eds.), Tasting and smelling (2nd ed., pp. 1-24). San Diego: Academic Press.

Breslin, P. A., \& Beauchamp, G. K. (1995). Suppression of bitterness by sodium: Variation among bitter taste stimuli. Chemical Senses, 20, 609-623.

CAicedo, A., \& Roper, S. D. (2001). Taste receptor cells that discriminate between bitter stimuli. Science, 291, 1557-1560.

Capeless, C. G., Whitney, G., \& Azen, E. A. (1992). Chromosome mapping of $S o a$, a gene influencing gustatory sensitivity to sucrose octaacetate in mice. Behavior Genetics, 22, 655-663.

Chandrashekar,J., Mueller, K. L., Hoon, M. A., Adler, E., Feng, L., Guo, W., Zuker, C. S., \& RYbA, N. J. P. (2000). T2Rs function as bitter taste receptors. Cell, 100, 703-711.

DAHL, M., ERICKSON, R. P., \& Simon, S. A. (1997). Neural responses to bitter compounds in rats. Brain Research, 756, 22-34.

FIscher, R. \& GRIFFIN, F. (1963). Quinine dimorphism: A cardinal determinant of taste sensitivity. Nature, 200, 343-347.

Frank, R. A., \& Korchmar, D. L. (1985). Gustatory processing differences in PTC tasters and non-tasters: A reaction time analysis. Physiology \& Behavior, 35, 239-242.

Frank, R. A., Van der Klaauw, N. J., \& Schifferstein, H. N. J. (1993). Both perceptual and conceptual factors influence taste-odor and taste-taste interactions. Perception \& Psychophysics, 54, 343-354.

Gent, J. F., \& BartoshuK, L. M. (1983). Sweetness of sucrose, neohesperidin dihydrochalcone, and saccharin is related to genetic ability to taste the bitter substance 6- $n$-propylthiouracil. Chemical Senses, 7, 265-272.

Green, B. G., Dalton, P., Cowart, B., Shaffer, G., Rankin, K., \& Higgins, J. (1996). Evaluating the "Labeled Magnitude Scale" for measuring sensations of taste and smell. Chemical Senses, 21, 323334. 
Green, B. G., Shaffer, G. S., \& Gilmore, M. M. (1993). Derivation and evaluation of a semantic scale of oral sensation magnitude with apparent ratio properties. Chemical Senses, 18, 683-702.

Guinard, J.-X., Hong, D. Y., Zoumas-Morse, C., Budwig, C., \& RusSELL, G. F. (1994). Chemoreception and perception of the bitterness of isohumulones. Physiology \& Behavior, 56, 1257-1263.

Hall, M. J., Bartoshuk, L. M., Cain, W. S., \& Stevens, J. C. (1975). PTC taste blindness and the taste of caffeine. Nature, 253, 442-443.

Harder, D. B., Boughter, J. D., \& Whitney, G. (1996). PTCavoidance polymorphism and other bitter-avoidance differences among mice in long-term preference tests. Chemical Senses, 21, 612.

Harder, D. B., Gannon, K. S., \& Whitney, G. (1996). SW.B6-Soa ${ }^{b}$ nontaster congenic strains completed and a sucrose octaacetate congenic quartet tested with other bitters. Chemical Senses, 21, 507-517.

Harder, D. B., \& Whitney, G. (1998). A common polygenic basis for quinine and PROP avoidance in mice. Chemical Senses, 23, 327-332.

Harder, D. B., Whitney, G., Frye, P., Smith, J. C., \& Rashotte, M. E. (1984). Strain differences among mice in taste psychophysics of sucrose octa-acetate. Chemical Senses, 9, 311-323.

Harris, H., \& Kalmus, H. (1949). Chemical specificity in genetical differences of taste sensitivity. Annals of Eugenics, 15, 32-45.

Jefferson, S. C., \& Erdman, A. M. (1970). Taste sensitivity and food aversions of teenagers. Journal of Home Economics, 62, 605-608.

Kemp, S. E., \& Birch, G. G. (1992). An intensity/time study of the taste of amino acids. Chemical Senses, 17, 151-168.

LAWLESS, H. T. (1979). The taste of creatine and creatinine. Chemical Senses \& Flavour, 4, 249-258.

Lawless, H. T., \& Clark, C. C. (1992). Psychological biases in timeintensity scaling. Food Technology, 46, 1992.

LeACH, E. J., \& Noble, A. C. (1986). Comparison of bitterness of caffeine and quinine by a time-intensity procedure. Chemical Senses, 11, 339-345.

Matsunami, H., Montmayeur, J.-P., \& Buck, L. B. (2000). A family of candidate taste receptors in human and mouse. Nature, 404, 601-604.

McBurney, D. H., Smith, D. V., \& Shick, T. R. (1972). Gustatory cross adaptation: Sourness and bitterness. Perception \& Psychophysics, 11, 228-232.

Mela, D. J. (1989). Bitter taste intensity: The effect of tastant and thiourea taster status. Chemical Senses, 14, 131-135.

Mela, D. J. (1990). Gustatory perception of isohumulones: Influence of sex and thiourea taster status. Chemical Senses, 15, 485-490.

Miller, I. J., JR., \& Whitney, G. (1989). Sucrose octaacetate-taster mice have more vallate taste buds than non-tasters. Neuroscience Letters, 100, 1-3.

O'Mahony, M. (1992). Understanding discrimination tests: A userfriendly treatment of response bias, rating and ranking R-index tests and their relationship to signal detection. Journal of Sensory Studies, 7, 1-47.

O’Mahony, M., Thieme, U., \& Goldstein, L. R. (1988). The warmup effect as a means of increasing the discriminability of sensory difference tests. Journal of Food Science, 53, 1848-1850.

Reed, D. R, Bartoshuk, L. M., Duffy, V., Marino, S., \& Price, R. A. (1995). Propylthiouracil tasting: Determination of underlying threshold distributions using maximum likelihood. Chemical Senses, 20, 529-533.

Schifferstein, H. N. J., \& FriJters, J. E. R. (1991). The perception of the taste of $\mathrm{KCl}, \mathrm{NaCl}$, and quinine $\mathrm{HCl}$ is not related to PROPsensitivity. Chemical Senses, 16, 303-317.

Schiffman, S. S., Gat lin, L. A., Frey, A. E., Heiman, S. A., Stagner, W. C., \& CoOper, D. C. (1994). Taste perception of bitter compounds in young and elderly persons: Relation to lipophilicity of bitter compounds. Neurobiology of Aging, 15, 743-750.

ShingaI, T., \& BeIDLER, L. M. (1985). Interstrain differences in bitter taste responses in mice. Chemical Senses, 10, 51-55.

Sмiтн, D. V. (1971). Taste intensity as a function of areas and concentration: Differentiation between compounds. Journal of Experimental Psychology, 87, 163-171.

Spielman, A. I., Huque, T., Whitney, G., \& Brand, J. G. (1992). The diversity of bitter taste signal transduction mechanisms. In D. P. Corey \& S. D. Roper (Eds.), Sensory transduction (pp. 308-324). New York: Rockerfeller University Press.

Thieme, U., \& O’Mahony, M. (1990). Modifications to sensory difference test protocols: The warmed up paired comparison, the single standard duo-trio and the A-not A test modified for response bias. Journal of Sensory Studies, 5, 159-176.

Thorngate, J. H., III (1997). Factors affecting the perception of bitterness: A review. In G. Roy (Ed.), Modifying bitterness: Mechanisms, ingredients, and applications (pp. 139-160). Valhalla, NY: Technomic Publishing.

Whitney, G., \& HARDER, D. B. (1994). Genetics of bitter perception in mice. Physiology \& Behavior, 56, 1141-1147.

Yokomukai, Y., Cowart, B. J., \& Beauchamp, G. K. (1993). Individual differences in sensitivity to bitter-tasting substances. Chemical Senses, 18, 669-681.

\section{APPENDIX \\ Calculation of Ranking R-Index Values}

The following is an illustration of how the R-index can be calculated for any two compounds from a set of nine that have been ranked from weakest to strongest in bitterness. First, create a response matrix that shows the number of times each compound received each rank (see Table A1). In Table A1, a lowercase letter that is used in the calculation formula identifies each cell.

The $\mathrm{R}$-index value is calculated by the following formula:

$$
\begin{aligned}
\text { R-index }= & 100 *\{[a(k+1+m+n+o+p+q+r)+b(1+m+n+o+p+q+r)+c(m+n+o+p+q+r)+ \\
& d(n+o+p+q+r)+e(o+p+q+r)+f(p+q+r)+g(q+r)+h(r)]+ \\
& 1 / 2[a j+b k+c l+d m+e n+f o+g p+h q+i r]\} / \\
& \{[a+b+c+d+e+f+g+h+i] *[j+k+1+m+n+o+p+q+r]\} .
\end{aligned}
$$

Table A1

Response Matrix for Calculating R-Index Values

\begin{tabular}{cccccccccr}
\hline & \multicolumn{10}{c}{ Rank } \\
\cline { 2 - 9 } Compound & 9 & 8 & 7 & 6 & 5 & 4 & 3 & 2 & 1 \\
\hline $\mathrm{X}$ & $\mathrm{a}$ & $\mathrm{b}$ & $\mathrm{c}$ & $\mathrm{d}$ & $\mathrm{e}$ & $\mathrm{f}$ & $\mathrm{g}$ & $\mathrm{h}$ & $\mathrm{i}$ \\
$\mathrm{Y}$ & $\mathrm{j}$ & $\mathrm{k}$ & $\mathrm{l}$ & $\mathrm{m}$ & $\mathrm{n}$ & $\mathrm{o}$ & $\mathrm{p}$ & $\mathrm{q}$ & $\mathrm{r}$ \\
\hline
\end{tabular}

Note-Rank is given in terms of strongest to weakest. 